\title{
Efeito do estádio de maturação e da temperatura na germinação de sementes de Sabal mauritiiformis ${ }^{(1)}$
}

\author{
PETTERSON BAPTISTA DA LUZ(2); RICARDO SOARES PIMENTA ${ }^{(3)}$ e KATHIA FERNANDES LOPES PIVETTA ${ }^{(4)}$
}

\begin{abstract}
RESUMO
Sabal mauritiiformis (H. Karst.) Griseb. ex. H. Wendl., originária da América Central e América do Sul, é uma palmeira que apresenta grande valor ornamental. A propagação comercial é sexuada e são poucos os estudos sobre a germinação de suas sementes, sendo estas influenciadas por fatores como o estádio de maturação e a temperatura. Este trabalho buscou avaliar o comportamento germinativo de sementes de $S$. mauritiiformis, a partir de frutos de três estádios de maturação, em diferentes temperaturas. O delineamento experimental foi o inteiramente casualizado, em esquema fatorial $3 \times 5$ ( 3 estádios de maturação e 5 condições de temperatura), com quatro repetições de 25 sementes. Frutos de coloração marrom, preto e verde foram despolpados e colocados para avaliação da capacidade germinativa das sementes sob as temperaturas de $25^{\circ} \mathrm{C}$, $30{ }^{\circ} \mathrm{C}, 35^{\circ} \mathrm{C}, 20-30^{\circ} \mathrm{C}$ e $25-35^{\circ} \mathrm{C}$, com fotoperíodo de 16 horas de luz e 8 horas de escuro, utilizando-se câmaras incubadoras tipo BOD. As sementes foram semeadas em vermiculita de granulometria média. Os resultados obtidos permitiram concluir que a temperatura de $30^{\circ} \mathrm{C}$ é a mais indicada para a germinação de sementes de Sabal mauritiiformis oriundas de frutos de coloração marrom, preto ou verde.
\end{abstract}

Palavras-chave: Arecaceae, palmeira, palmeira-leque, propagação.

\begin{abstract}
Effect of maturation stage and temperature on the germination of sabal mauritiiformis seeds Sabal mauritiiformis (H. Karst.) Griseb. ex. H. Wendl. is a palm tree native from Central America and South America showing high ornamental value. Its commercial propagation is done sexual. There are few studies on seed germination under the influence of various factors such as maturation stage and temperature. This work to evaluate the behavior of seed germination of $S$. mauritiiformis, utilizing fruits of different maturation stages under different temperatures. The experiment was carried out in a completely randomized on a 3 x 5 factorial design (three maturation stages and five temperature conditions), with four replicates of 25 seeds each. Brownish, black and greenish fruits were depulped and their germinative capacity was evaluated under temperatures of $25^{\circ} \mathrm{C}, 30^{\circ} \mathrm{C}, 35^{\circ} \mathrm{C}, 20-30{ }^{\circ} \mathrm{C}$ e $25-35^{\circ} \mathrm{C}$, and photoperiod of $16 \mathrm{~L}$ :8D, using BOD incubation chambers with controlled temperature and photoperiod. The seeds were sowed on vermiculite. The results show that the temperature of $30^{\circ} \mathrm{C}$ was the most indicated for the germination of Sabal mauritiiformis seeds coming from both the brownish, black and greenish fruits.
\end{abstract}

Keywords: Arecaceae, palm tree, fan palm, propagation.

\section{INTRODUÇ̃̃O}

A palmeira Sabal mauritiiformis (H. Karst.) Griseb. ex. H. Wendl., originária da América Central e América do Sul, é rústica, adaptando-se a diversos climas e solos, resistindo à seca e aos ventos marinhos. É uma palmeira solitária, monóica, esguia e com caule liso, verde na juventude e depois pardo, anelado e com uma dilatação distinta na base. Embora apresente características ornamentais notáveis, não é muito difundida, no Brasil (LORENZI et al., 2004). Na literatura, há poucas informações sobre propagação dessa espécie.

A propagação comercial da maioria das palmeiras é por sementes e, segundo Meerow (1991), de modo geral, a germinação é lenta, desigual e influenciada por fatores como o estádio de maturação para a colheita dos frutos e a temperatura durante o processo de germinação.
A determinação da época de colheita das sementes de uma espécie pode ser facilitada pela adoção de índices de maturação visíveis no aspecto externo dos frutos e das sementes (PINA-RODRIGUES e AGUIAR, 1993).

Para as palmeiras, Lorenzi et al. (2004) indicam que os melhores resultados de germinação são obtidos com sementes provenientes de frutos maduros, sendo a germinação de sementes de frutos imaturos muito falha, podendo até não ocorrer, porque o endosperma se encontra ainda aquoso, não solidificado. Os mesmos autores reportaram que os frutos de palmeira têm grande variação de cor, não raro passam por diversas tonalidades durante o amadurecimento.

Iossi (2005), trabalhando com a palmeira Phoenix roebelenii, correlacionou dados biométricos, teor de água das sementes, dias após antese e coloração para determinar o

\footnotetext{
(1) Trabalho recebido para publicação em 30/03/2012 e aprovado em 30/05/2014;

(2) Engenheiro Agrônomo, Professor Dr. em Produção e Tecnologia de Sementes - Departamento de Agronomia - Universidade do Estado de Mato Grosso/ UNEMAT - Av. São João, s/nº - Cavalhada - CEP 78200-000 Cidade: Cáceres / MT - e-mail: petterbaptista@yahoo.com.br;

(3) Biológo, Doutorando em Produção e Tecnologia de Sementes Departamento de Produção Vegetal Universidade Estadual Paulista/UNESP Via de Acesso Professor Paulo Donato Castellane, s/n 14884-900 Jaboticabal, SP pimenta@fcav.unesp.br;

(4) Professora Doutora. Departamento de Produção Vegetal - UNESP/FCAV. Via de acesso Prof. Paulo Donato Castellane, s/n, 14884-900, Jaboticabal, SP. E-mail:kathia@fcav.unesp.br.
} 
ponto de maturidade fisiológica, no entanto, essa análise se mostra pouco prática, e que a coloração dos frutos não é um bom indicador do ponto de maturidade fisiológica das sementes. O mesmo autor, porém, ressalta que a coloração preto-violácea dos frutos de $P$. roebelenii é um bom parâmetro para revelar o período adequado de colheita dos frutos.

Para Syagrus schizophylla, Pivetta et al. (2005c) concluíram que os melhores resultados foram obtidos com frutos de coloração amarelos ou avermelhados. Sementes de Livistona rotundifolia apresentaram germinação semelhante tanto para frutos colhidos verdes como maduros, de acordo com Viana (2003). Já os melhores resultados obtidos para Livistona chinensis foram a partir de frutos colhidos verdes (MACIEL, 1996).

De acordo com Carvalho e Nakagawa (2000), a temperatura influencia tanto a velocidade quanto a uniformidade de germinação, sendo as sementes capazes de germinar sob determinada amplitude de temperatura, definida para cada espécie, existindo uma temperatura máxima e uma mínima. Na Família Arecaceae, há indicações de temperaturas ideais para germinação de sementes que variam desde $24{ }^{\circ} \mathrm{C}$ até $35^{\circ} \mathrm{C}$ (MEEROW, 1991; BROSCHAT, 1994; LORENZI et al., 2004).

Para Meerow (1991), temperaturas entre $20{ }^{\circ} \mathrm{C}$ e 40 ${ }^{\circ} \mathrm{C}$ são aceitáveis para a germinação de sementes de palmeiras, porém os melhores resultados são obtidos com temperaturas entre $30{ }^{\circ} \mathrm{C}$ e $35^{\circ} \mathrm{C}$, para a maior parte das espécies.

Embora a maioria das palmeiras seja de origem tropical e as sementes geralmente germinam naturalmente em temperaturas mais elevadas, muitos trabalhos testando temperaturas variadas evidenciaram que maior porcentagem de germinação têm sido encontrada em diferentes espécies, como $35{ }^{\circ} \mathrm{C}$ para Acoelorraphe wrightii, Coccothrinax argentata, Sabal etonia, Thrinax morrisii, Thrinax parviflora e Roystonea regia (CARPENTER, 1988; PIVETTA et al., 2005a; PENARIOL, 2005), $25^{\circ} \mathrm{C}$ e 30 ${ }^{\circ} \mathrm{C}$ para Phoenix roebelenii (IOSSI et al., 2003), $25{ }^{\circ} \mathrm{C}$ e alternada $20-30{ }^{\circ} \mathrm{C}$ para Rhapis excelsa (AGUIAR et al., 2005; LUZ et al., 2008), $30{ }^{\circ} \mathrm{C}$ e $35^{\circ} \mathrm{C}$ para Syagrus romanzoffiana (PIVETTA et al., 2005b) e temperaturas alternadas de $30-35^{\circ} \mathrm{C}$ para Chrysalidocarpus lutescens (BROSCHAT e DONSELMAN, 1986), 20-30 ${ }^{\circ} \mathrm{C}$ e $25^{\circ} \mathrm{C}$ para Euterpe edulis (ANDRADE et al., 1999) e $25-35^{\circ} \mathrm{C}$ para Livistona rotundifolia (VIANA, 2003).

Alguns estudos correlacionaram temperatura e estádio de maturação, sendo este definido pela coloração externa do fruto. Penariol (2005) analisou três tonalidades do fruto (vermelho, amarelo e preto) e seis condições de temperatura (constante de $20{ }^{\circ} \mathrm{C}, 25{ }^{\circ} \mathrm{C}, 30{ }^{\circ} \mathrm{C}, 35^{\circ} \mathrm{C}$ e alternadas de $20-30{ }^{\circ} \mathrm{C}$ e $25-35^{\circ} \mathrm{C}$ ) e concluiu que a maior porcentagem $(99,7 \%)$ e a maior velocidade de germinação das sementes foram obtidas por aquelas provenientes de frutos pretos germinadas em temperatura de $35^{\circ} \mathrm{C}$.

Devido à importância e à falta de informações na literatura sobre a propagação desta espécie, este trabalho teve como objetivo estudar o efeito do estádio de maturação e da temperatura na germinação de sementes de Sabal mauritiiformis.

\section{MATERIAL E MÉTODOS}

Foram coletados frutos de Sabal mauritiiformis em diferentes estádios de maturação estádio 1 (fruto inteiramente verde, massa seca do fruto de $0,97 \mathrm{~g}$ ), estádio 2 (fruto marron, massa seca do fruto de $0,97 \mathrm{~g}$ ) e estádio 3 (fruto inteiramente preto, massa seca do fruto de $1,00 \mathrm{~g}$ ), de seis matrizes na cidade de Jaboticabal-SP (21 $\left.{ }^{\circ} \mathrm{S}, 48^{\circ} \mathrm{W}\right)$, Classificação Climática de Koeppen: Aw.

Após a colheita, os frutos foram separados, manualmente, dos cachos, e agrupados de acordo com as colorações mencionadas. Em seguida, foram imersos em água por 24 horas, para posterior retirada do epicarpo e mesocarpo. Os diásporos (sementes + complexo orgânico, no caso endocarpo) obtidos foram secos à sombra por 24 horas.

Os diásporos foram colocados em caixas plásticas transparentes com tampa tipo "gerbox", nas dimensões de 11 x 11 × $3 \mathrm{~cm}$, utilizando vermiculita de granulometria média como substrato. As caixas foram colocadas em germinadores com temperaturas reguladas de acordo com o tratamento, fotoperíodo de 8 horas de luz (geradas por duas lâmpadas fluorescentes) e 16 horas de escuro. O teste foi conduzido durante 70 dias, quando não mais se observou a germinação de sementes, verificada pela emissão do botão germinativo.

O delineamento experimental foi o inteiramente casualizado em esquema fatorial 3 x 5 (3 estádios de maturação caracterizados pela coloração externa dos frutos: marrom, preto e verde e 5 condições de temperatura: constantes de $25^{\circ} \mathrm{C}, 30^{\circ} \mathrm{C}, 35^{\circ} \mathrm{C}$ e alternadas $(12 \mathrm{~h} / 12 \mathrm{~h})$ de $20-30{ }^{\circ} \mathrm{C}$ e $25-35^{\circ} \mathrm{C}$, com quatro repetições de 25 sementes.

A porcentagem de germinação foi calculada pela fórmula proposta nas Regras para Análise de Sementes (BRASIL, 2009) e o índice de velocidade de germinação calculado de acordo com a fórmula proposta por Maguire (1962).

Os dados foram submetidos à análise de variância, sendo que os de porcentagem de germinação foram previamente transformados em arcsen $\sqrt{x} / 100$ e analisados estatisticamente com auxílio do programa SISVAR ${ }^{\circledR}$ (FERREIRA, 2011); as médias comparadas pelo teste de Scott-Knott a $5 \%$ de probabilidade.

\section{RESULTADOS E DISCUSSÃO}

Não houve diferença significativa para os diferentes estádios de maturação dos frutos quanto à porcentagem de germinação das sementes de $S$. mauritiiformis.

Tanto para a porcentagem, quanto para o IVG, a interação entre temperatura e estádio de maturação foi significativa (Tabela 1). As sementes provenientes de frutos marrons, pretos e verdes apresentaram menor porcentagem de germinação na temperatura de $35^{\circ} \mathrm{C}$ e germinam mais rápido na temperatura de $30{ }^{\circ} \mathrm{C}$. A condição que permitiu maior porcentagem de germinação das sementes de $S$. mauritiiformis foi com frutos de coloração verde, ou seja, no estádio de maturação menos avançado, na temperatura constante de $30{ }^{\circ} \mathrm{C}$ e alternada de $20-30{ }^{\circ} \mathrm{C}$, atingindo as maiores taxas de germinação $(97 \%)$ e IVG, porém não diferindo estatisticamente da temperatura constante de $25^{\circ} \mathrm{C}$ e alternadas de $25-35^{\circ} \mathrm{C}$. 
Tabela 1. Quadrados médios e médias obtidas para porcentagem de germinação e Índice de Velocidade de Germinação (IVG) de sementes de Sabal mauritiiformis submetidas a 3 estádios de maturação e 5 diferentes temperaturas. Jaboticabal, SP, 2006.

Table 1. Averages and averages squares of germination percentage and speed of germination index (IVG) of Sabal mauritiiformis seeds submitted to 3 maturation stadiums and 5 different temperatures. Jaboticabal, SP, 2006.

\begin{tabular}{|c|c|c|c|}
\hline Fontes de Variação & GL & Porcentagem de germinação ${ }^{1}$ & $\mathrm{IVG}^{2}$ \\
\hline Estádio de maturação (E) & 1 & $13,3447^{\mathrm{NS}}$ & $0,2351 * *$ \\
\hline Temperatura $(\mathrm{T})$ & 4 & $1190,7964 * *$ & $0,8160 * *$ \\
\hline $\mathrm{E} \times \mathrm{T}$ & 4 & $153,2444 *$ & $0,0310 * *$ \\
\hline Repetição & 3 & 73,7662 & 0,0027 \\
\hline Resíduo & 27 & 70,9771 & 0,0094 \\
\hline $\mathrm{CV}(\%)$ & & 11,52 & 12,04 \\
\hline \multicolumn{4}{|c|}{ Médias } \\
\hline Marrom $-25^{\circ} \mathrm{C}$ & & $78,90^{1}(95,00)^{2} \mathrm{a}$ & $0,9897 \mathrm{~b}$ \\
\hline Marrom $-30^{\circ} \mathrm{C}$ & & $78,90(95,00) \mathrm{a}$ & $1,2255 \mathrm{a}$ \\
\hline Marrom $-35^{\circ} \mathrm{C}$ & & $61,65(77,00) b$ & $0,3427 \mathrm{c}$ \\
\hline Marrom $-20-30^{\circ} \mathrm{C}$ & & $72,04(90,00) \mathrm{a}$ & $0,9875 \mathrm{~b}$ \\
\hline Marrom $-25-35^{\circ} \mathrm{C}$ & & $73,00(91,00) \mathrm{a}$ & $1,0126 \mathrm{~b}$ \\
\hline Preto $-25^{\circ} \mathrm{C}$ & & $80,47^{1}(94,00)^{2} \mathrm{a}$ & $0,8779 \mathrm{~b}$ \\
\hline Preto $-30^{\circ} \mathrm{C}$ & & $79,16(95,00) \mathrm{a}$ & $1,0750 \mathrm{a}$ \\
\hline Preto $-35^{\circ} \mathrm{C}$ & & $61,65(77,00) b$ & $0,4899 \mathrm{c}$ \\
\hline Preto $-20-30^{\circ} \mathrm{C}$ & & $69,03(87,00) b$ & $0,7557 \mathrm{~b}$ \\
\hline Preto $-25-35^{\circ} \mathrm{C}$ & & $79,99(94,00) \mathrm{a}$ & $0,8977 \mathrm{~b}$ \\
\hline Verde $-25^{\circ} \mathrm{C}$ & & $79,99^{1}(94,00)^{2} \mathrm{a}$ & $0,7581 \mathrm{a}$ \\
\hline Verde $-30^{\circ} \mathrm{C}$ & & $81,34(97,00) \mathrm{a}$ & $0,8423 \mathrm{a}$ \\
\hline Verde $-35^{\circ} \mathrm{C}$ & & $44,30(49,00) b$ & $0,2550 \mathrm{~b}$ \\
\hline Verde $-20-30^{\circ} \mathrm{C}$ & & $81,34(97,00) \mathrm{a}$ & $0,8135 \mathrm{a}$ \\
\hline Verde $-25-35^{\circ} \mathrm{C}$ & & $75,45(93,00) \mathrm{a}$ & $0,8086 \mathrm{a}$ \\
\hline
\end{tabular}

As médias seguidas da mesma letra não diferem entre si pelo teste de Scott Knott a 5\% de probabilidade.

${ }^{1}$ Dados transformados em arc. sen. $\sqrt{ } \mathrm{x} / 100$.

${ }^{2}$ Dados não transformados.

* Significativo a $5 \%$ de probabilidade, $* *$ Significativo a $1 \%$ de probabilidade.

Estes resultados vão contra os comentários de Pivetta et al. (2007) de que é recomendado colher sementes de palmeira quando estiverem bem maduros e, neste caso, pelos resultados obtidos, sementes de $S$. mauritiiformis atingiram o ponto de maturidade fisiológica quando os frutos ainda estavam com a coloração verde.

Esses resultados sugerem que não houve deterioração das sementes de uma fase de coloração para a outra. Considerando-se que em um mesmo cacho encontram-se frutos das três categorias e, portanto, não há como realizar colheita seletiva de frutos pelo grau de maturação, após a colheita dos cachos não é necessário fazer a separação das sementes para a produção de mudas.

Os dados obtidos nesse estudo, aliados aos da literatura, reforçam que o ponto ideal de colheita de sementes de palmeiras pode ocorrer antes do completo amadurecimento dos frutos e que a mudança de coloração externa dos mesmos presta-se como indicativo de maturidade das sementes e não pode ser generalizada para a família Arecaceae. 
Também Chapin (1999), estudando a maturação dos frutos de oito espécies de palmeiras, utilizou a coloração do epicarpo como um dos critérios de indicação de fruto maduro.

Aguiar et al. (2005) também estudaram o efeito da temperatura e do estádio de maturação na germinação de sementes da palmeira Rhapis excelsa, na qual os melhores resultados foram com frutos em estádio de maturação amarelo (intermediário) na temperatura de $25^{\circ} \mathrm{C}$.

$\mathrm{O}$ efeito da temperatura na germinação de sementes de várias espécies de palmeiras foi estudado por Andrade et al. (1999), Iossi et al. (2003), Pivetta et al. (2005a),

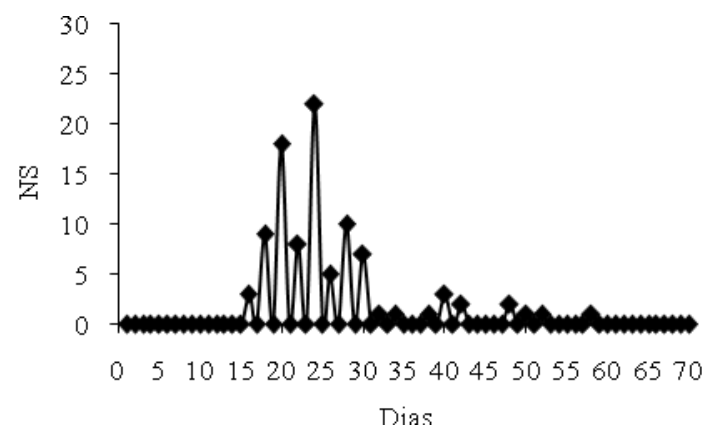

$25^{\circ} \mathrm{C}$

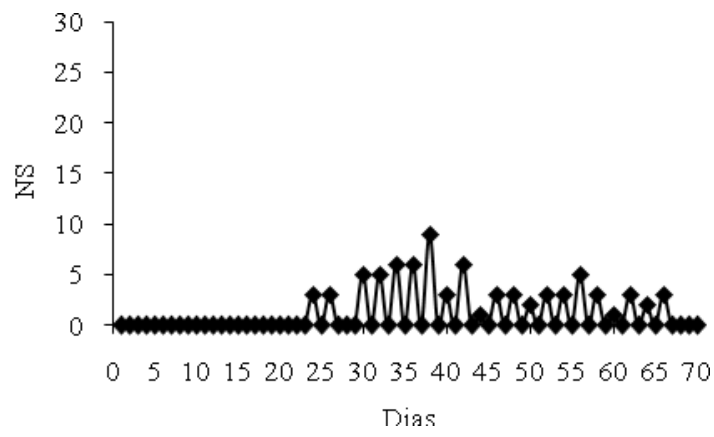

$35^{\circ} \mathrm{C}$

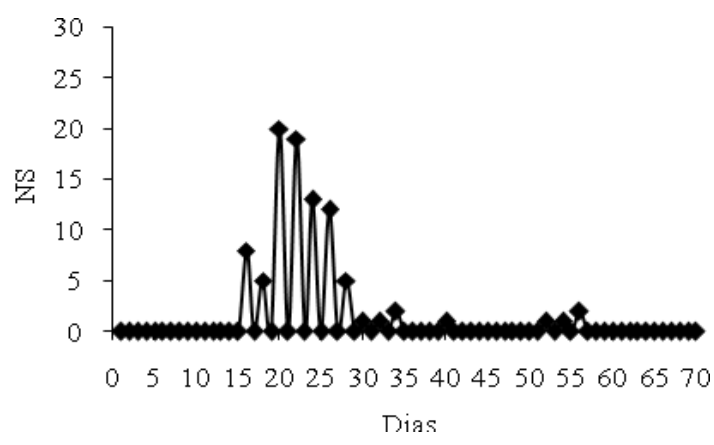

25-35 ${ }^{\circ} \mathrm{C}$
Pivetta et al. (2005b) e Luz et al. (2008), porém não fizeram correlações com o estádio de maturação.

As diferentes faixas de temperaturas empregadas influenciaram diferentemente nos três estádios de maturação; entretanto, a temperatura fixa de $35^{\circ} \mathrm{C}$ se mostrou inadequada para o processo de germinação, apresentando as menores médias de porcentagem de germinação e IVG.

As Figuras 1, 2 e 3 mostram que a germinação de sementes de $S$. mauritiiformis foi heterogênea, reforçando os comentários de Meerow (1991) que, de modo geral, a germinação de sementes de palmeira é lenta e desigual.

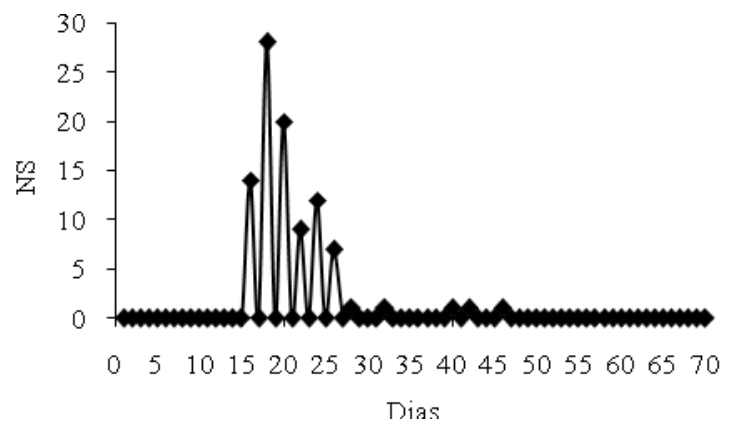

$30{ }^{\circ} \mathrm{C}$

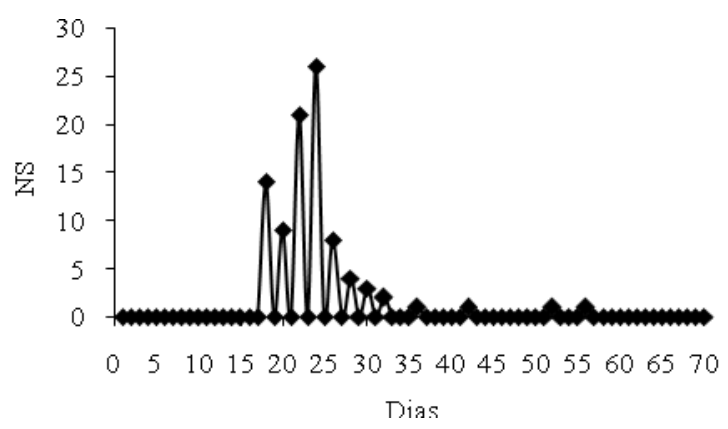

20-30 ${ }^{\circ} \mathrm{C}$

Figura 1. Distribuição da germinação de sementes (NS - número de sementes germinadas dia-1) ao longo de 100 dias, de um lote de 100 sementes de Sabal mauritiiformis em diferentes condições de temperatura e estádio de maturação marrom. Jaboticabal, SP, 2006.

Figure 1. Distribution of seeds germination (NS - number of germinated seeds day-1) during 100 days, from one lot of 100 seeds of Sabal mauritiiformis in different conditions of temperature and brownish stadium of maturation. Jaboticabal, SP, 2006. 


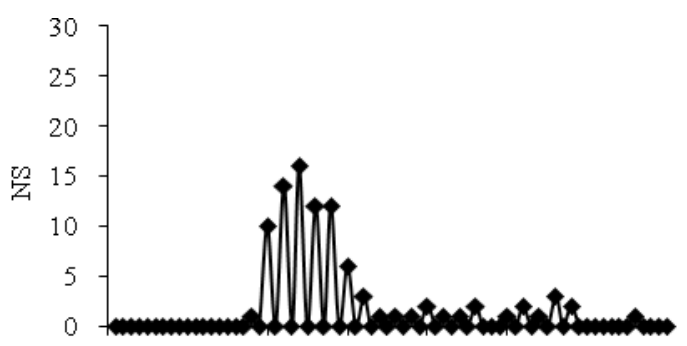

$0 \quad 510152025303540455055606570$

Dias

$25^{\circ} \mathrm{C}$

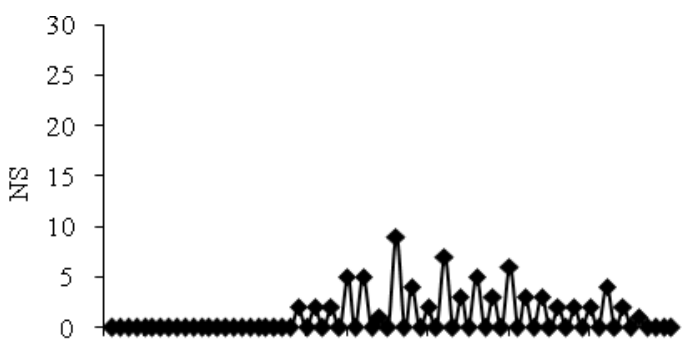

$0 \quad 510152025303540455055606570$ Dias

$35^{\circ} \mathrm{C}$

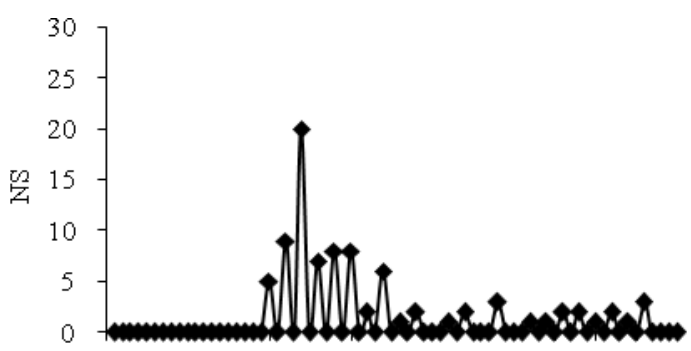

$0 \quad 5 \quad 10152025303540455055606570$

Dias

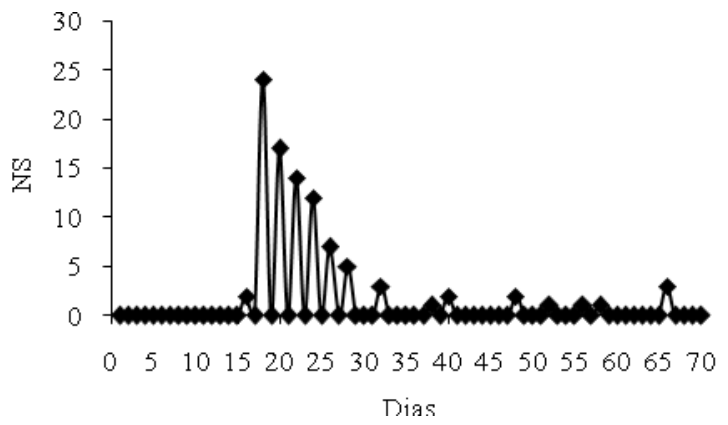

$30{ }^{\circ} \mathrm{C}$

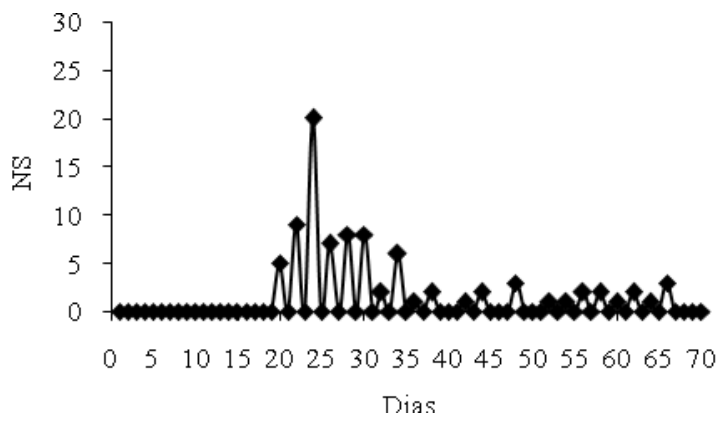

20-30 ${ }^{\circ} \mathrm{C}$

25-35 ${ }^{\circ} \mathrm{C}$

Figura 2. Distribuição da germinação de sementes (NS - número de sementes germinadas dia-1) ao longo de 100 dias, de um lote de 100 sementes de Sabal mauritiiformis em diferentes condições de temperatura e estádio de maturação preto. Jaboticabal, SP, 2006.

Figure 2. Distribution of seeds germination (NS - number of germinated seeds day-1) during 100 days, from one lot of 100 seeds of Sabal mauritiiformis in different conditions of temperature and black stadium of maturation. Jaboticabal, SP, 2006. 


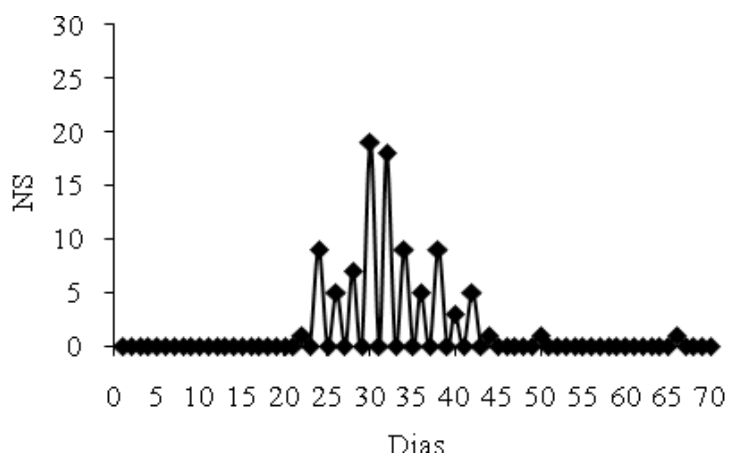

$25^{\circ} \mathrm{C}$

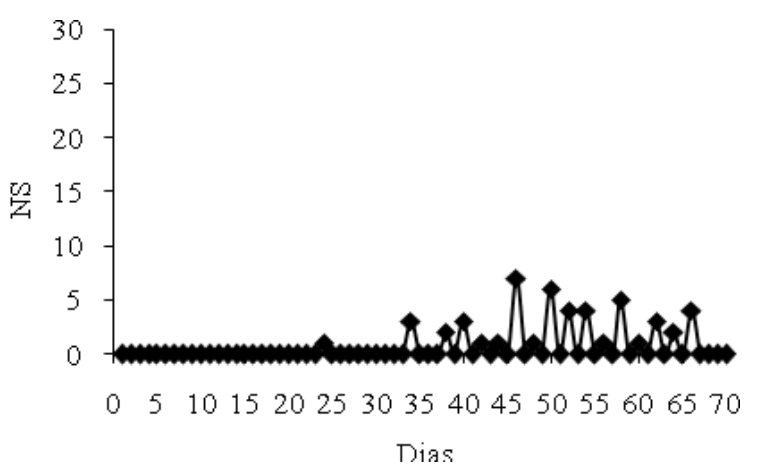

$35^{\circ} \mathrm{C}$

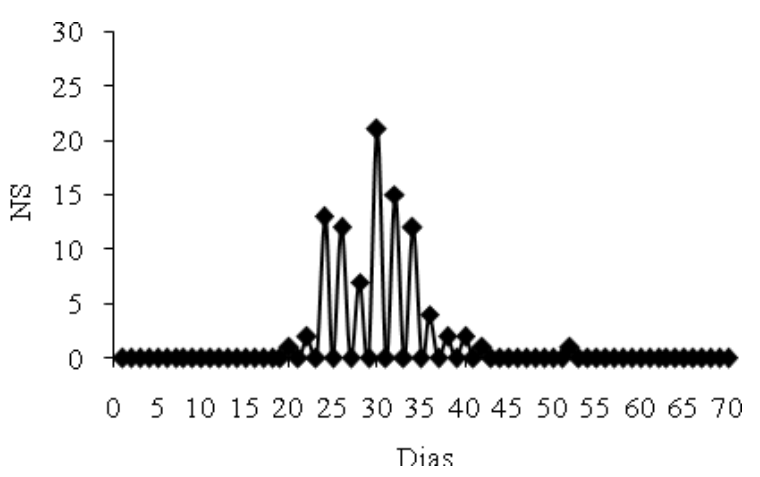

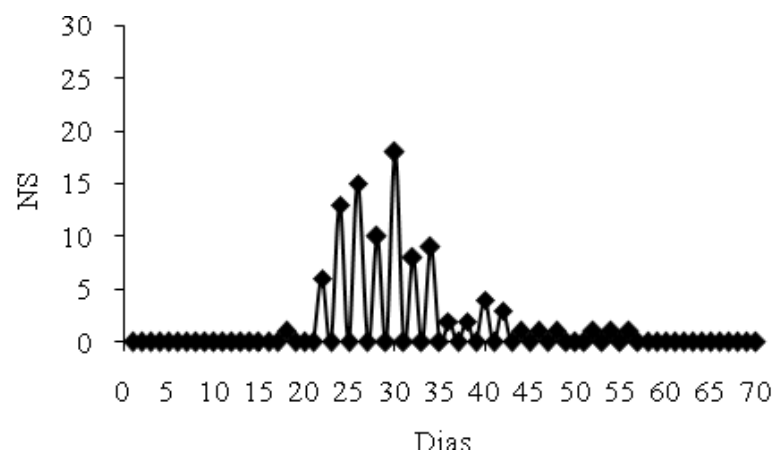

$30{ }^{\circ} \mathrm{C}$

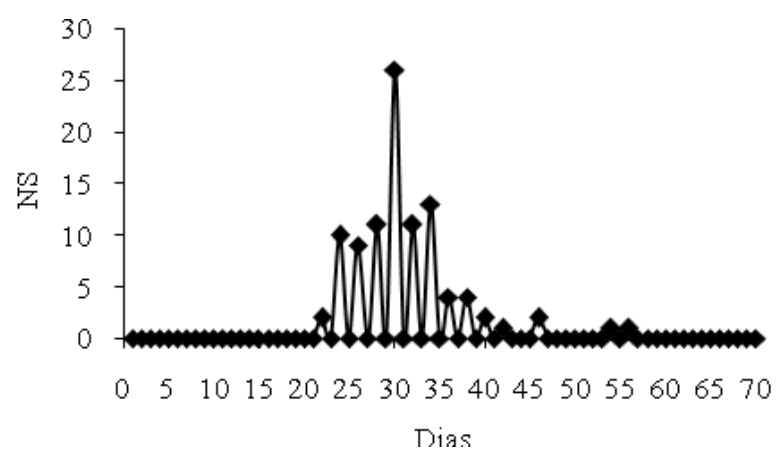

20-30 ${ }^{\circ} \mathrm{C}$

\section{$25-35^{\circ} \mathrm{C}$}

Figura 3. Distribuição da germinação de sementes (NS - número de sementes germinadas dia-1) ao longo de 100 dias, de um lote de 100 sementes de Sabal mauritiiformis em diferentes condições de temperatura e estádio de maturação verde. Jaboticabal, SP, 2006.

Figure 3. Distribution of seeds germination (NS - number of germinated seeds day-1) during 100 days, from one lot of 100 seeds of Sabal mauritiiformis in different conditions of temperature and greenish stadium of maturation. Jaboticabal, SP, 2006.

A germinação das sementes provenientes de frutos marrons (Figura 1) iniciou por volta do décimo quinto dia para as temperaturas de $25^{\circ} \mathrm{C}, 30^{\circ} \mathrm{C}, 20-30{ }^{\circ} \mathrm{C}$ e $25-35^{\circ} \mathrm{C}$ e no vigésimo quarto dia para a temperatura de $35^{\circ} \mathrm{C}$. Para sementes provenientes de frutos pretos (Figura 2), a germinação iniciou no décimo oitavo dia para as temperaturas supra citadas, e no vigésimo terceiro dia para a temperatura de 35 ${ }^{\circ} \mathrm{C}$. Já para aquelas ou aqueles se for usar diásporos provenientes de frutos verdes (Figura 3), a germinação iniciou em torno do décimo oitavo dia para as mesmas temperaturas e no vigésimo quinto dia para a temperatura de $35^{\circ} \mathrm{C}$.

$\mathrm{O}$ pico de germinação foi variável nas diferentes temperaturas, no entanto, a grande maioria das sementes, inde- pendentemente da temperatura, germinou do décimo quinto ao quadragésimo dia, mostrando que, embora a germinação tenha sido desuniforme, foi rápida quando comparada com outras espécies da família Arecaceae, como Euterpe edulis, cuja germinação finalizou aos 98 dias, ou seja, 14 semanas (ANDRADE et al., 1999).

Andrade et al. (1999) comentaram sobre a importância de padronizar os métodos de análise de sementes para as espécies que não constam nas Regras para Análise de Sementes (BRASIL, 2009) e, baseados nos gráficos de germinação ao longo do tempo, indicaram a sétima e a décima quarta semanas como contagem inicial e final nos testes de germinação de sementes de Euterpe edulis. 
Dessa forma, considerando o melhor resultado obtido neste estudo (frutos nos três estádios de maturação desde que não submetidos a germinação na temperatura constante de $35^{\circ} \mathrm{C}$ ), há indicativo de contagens inicial e final, respectivamente, no décimo oitavo e $55^{\circ}$ dia.

\section{CONCLUSÃO}

A temperatura de $30{ }^{\circ} \mathrm{C}$ é a mais indicada para a germinação de sementes de Sabal mauritiiformis oriundas de frutos de coloração marrom, preto e verde.

\section{AGRADECIMENTOS}

Os autores agradecem a Fapesp pelo auxílio pesquisa.

\section{REFERÊNCIAS}

AGUIAR, F. F. A.; BILIA, D. A. C.; KANASHIRO, S.; TAVARES, A. R.; BARBEDO, C. J. Germinação de sementes de Rhapis excelsa (Thunb.) Henry ex Rehder: efeitos da temperatura, luz e substrato. Hoehnea, São Paulo, v.32, n.1, p.119-126, 2005.

ANDRADE, A. C. S.; LOUREIRO, M. B.; SOUZA, A. D. O; RAMOS, F. N.; CRUZ, A. P. N. Reavaliação do efeito do substrato e da temperatura na germinação de sementes de palmiteiro (Euterpe edulis Mart.). Revista Árvore, v.23, n.3, p.279-283, 1999.

BRASIL. Ministerio da Agricultura, Pecuaria e Abastecimento. Regras para análise de sementes. Brasilia: Mapa/ ACS, 2009. 399p.

BROSCHAT, T. K. Palm seed propagation. Acta Horticulturae, n.360, p.141-147, 1994.

BROSCHAT, T. K.; DONSELMAN, H. Factors affecting storage and germination of Chrysalidocarpus lutescens seeds. Journal of American Society of Horticultural Science, v.111, n.6, p.872-877, 1986.

CARPENTER, W. J. Temperature affects seed germination of four Florida palm species. HortScience, v.23, n.2, p.336-337, 1988.

CARVALHO, N. M.; NAKAGAWA, J. Sementes: ciência, tecnologia e produção. 4.ed. Jaboticabal: Funep, 2000. $588 \mathrm{p}$.

CHAPIN, M. H. Flowering and fruiting phenology in certain palms. Palms, v.43, n.4, p.161-165, 1999.

FERREIRA, D. F. Sisvar: a computer statistical analysis system. Ciência e Agrotecnologia, Lavras, v. 35, n. 6, p. 1039-1042, nov./dez. 2011

IOSSI, E.; SADER, R.; PIVETTA, K. F. L.; BARBOSA, J. C. Efeitos de substratos e temperaturas na germinação de sementes de tamareira-anã (Phenix roebelenii O'Brien). Revista Brasileira de Sementes, v.25, p.63-69, 2003.
IOSSI, E. Morfologia floral e maturação fisiológica de sementes de tamareira-anã (Phoenix roebelenii O'Brien) Arecaceae. (Doutorado em Produção Vegetal). Faculdade de Ciências Agrárias e Veterinárias, Universidade Estadual Paulista, Jaboticabal, 2005. $51 \mathrm{f}$.

LORENZI, H.; SOUZA, H. M.; COSTA, J. T. M.; CERQUEIRA, L. S. C.; FERREIRA, E. Palmeiras brasileiras exóticas e cultivadas. Nova Odessa: Instituto Plantarum, 2004, 416 p.

LUZ, P. B.; PIMENTA, R. S.; PIZETTA, P. U. C.; CASTRO, A.; PIVETTA, K. F. L. Germinação de sementes de Dypsis decaryi (Jum.) Beentje \& J. Dransf. (Arecaceae). Ciência e Agrotecnologia, v.32, n.5, p.1461-1466, 2008.

LUZ, P. B.; TAVARES, A. R.; PAIVA, P. D. O.; AGUIAR, F. F. A.; KANASHIRO, S. Germinação de sementes de palmeira-ráfia: efeito de tratamentos pré-germinativos. Revista Árvore, vol.32, n.5, p.793-798, 2008.

MACIEL, N. M. S. Efectos de la madurez y el almacenamiento del fruto, la escarificacion y el remojo de las semillas sobre la emergencia de la palma china de abanico. Agronomia Tropical, v.46, n.2, p.155-170, 1996.

MAGUIRE, J. D. Speed of germination aid in selection and evaluation for seedling emergence and vigor. Crop Science, v.2, n.2, p.176-177, 1962.

MEEROW, A. W. Palm seed germination. Florida: Cooperative Extension Service, 1991. 10p. (Bulletin 274)

PENARIOL, A. P. Efeito da temperatura e do estádio de maturação na germinação de sementes de Roystonea regia (Kunth) O.F. Cook (Arecaceae). 2005. 32f. Monografia (Trabalho de Graduação em Agronomia) - Faculdade de Ciências Agrárias e Veterinárias, Universidade Estadual Paulista, Jaboticabal, 2005.

PIÑA-RODRIGUES, F. C. M.; AGUIAR, I. B. Maturação e dispersão de sementes. In: I. B. Aguiar, F. C. M. Piña-Rodrigues, M. B. Figliolia (Eds.). Sementes Florestais Tropicais. Brasília: Associação Brasileira de Tecnologia de Sementes, p. 215-274, 1993.

PIVETTA, K. F. L.; CASALI, L. P.; CINTRA, G. S.; PEDRINHO, D. R.; PIZETTA, P. U. C.; PIMENTA, R. S.; PENARIOL, A. P.; MATTIUZ, C. F. M. Efeito da temperatura e do armazenamento na germinação de sementes de Thrinax parviflora Swartz. (Arecaceae). Científica, Jaboticabal, v.33, n.2, p.178-184, 2005a.

PIVETTA, K. F. L.; BARBOSA, J. G.; ARAÚJO, E. F.; DEMATTÊ, M. E. E. P. Propagação de palmeiras e estrelitzias. In: BARBOSA, J. G.; LOPES, L. C. Propagação de plantas ornamentais. Viçosa, Universidade Federal de Viçosa, p.43-70, 2007. 
PIVETTA, K. F. L.; PAULA, R. C.; CINTRA, G. S.; PEDRINHO, D. R.; CASALI, L. P.; PIZETTA, P. U. C.; PIMENTA, R. S. Effects of temperature on seed germination of Queen Palm Syagrus romanzoffiana (Cham.) Glassman. (Arecaceae). Acta Horticulturae, Leuven, v.683, p.379-381, 2005b.

PIVETTA, K. F. L.; PAULA, R. C.; CINTRA, G. S.; PEDRINHO, D. R.; CASALI, L. P.; PIZETTA, P. U. C.; SARZI, I.; PIMENTA, R. S. Effects of maturation and scarification on seed germination of Syagrus schizophylla (Mart.) Glass. (Arecaceae). Acta Horticulturae, Wageningen, v.683, p.375-378, 2005c.

VIANA, F. A. P. Estudos sobre germinação e morfo-anatomia do diásporo e da plântula de Livistona rotundifolia (Lam.) Mart. (Arecaceae). 2003. 71f. Dissertação (Mestrado em Produção e Tecnologia de Sementes) - Faculdade de Ciências Agrárias e Veterinárias, Universidade Estadual Paulista, Jaboticabal, 2003. 